\title{
Iris crystals in chronic uveitis
}

\author{
Andrew B Callear, Anita Reynolds, John Harry, Philip I Murray
}

\begin{abstract}
Aims-To analyse the unusual physical sign of iris crystals occurring in patients with uveitis.

Methods-Demographic details and clinical features were documented in 24 patients with chronic uveitis and iris crystals. Plasma immunoglobulin subclasses were measured, and a histopathological review of iridectomy specimens from 33 patients with chronic uveitis was also undertaken.

Results-The mean age of patients was 38 years, with a slight preponderance of females. 17 patients had Fuchs' heterochromic cyclitis although a number of other uveitis entities were represented. There was no correlation between severity of clinical signs and presence of iris crystals. Over a mean follow up period of 15 months no significant change in the number, size, or position of the crystals was seen except in four patients who underwent intraocular surgery. Only three patients had raised plasma IgG1. The review of the histology of iridectomy specimens failed to show evidence of Russell body formation in any patient.

Conclusions-Iris crystals appear to be rare but may be underreported as they are small and can easily be missed. They are likely to be associated with disease processes in which there is active immunoglobulin production within the anterior chamber, such as Fuchs' heterochromic cyclitis.
\end{abstract}

(Br f Ophthalmol 1999;83:703-706)
The aetiology of the majority of cases of chronic uveitis remains obscure but they are thought to represent a $\mathrm{T}$ lymphocyte mediated disease. ${ }^{1}$ Nevertheless, B lymphocytes and plasma cells are often found within the iris stroma and raised immunoglobulin (Ig) levels associated with oligoclonal Ig bands have been reported in the aqueous of patients with Fuchs' heterochromic cyclitis (FHC). ${ }^{2}{ }^{3}$

The clinical signs which characterise intraocular inflammation are well known and include keratic precipitates, flare, cells, and posterior synechiae. Crystals on the iris of patients with chronic uveitis appear to be a rare clinical sign. ${ }^{4-10}$ They are seen as tiny refractile deposits within the iris stroma on slit lamp examination. They may occur anywhere from the pupil margin to the peripheral iris. Careful scanning of the iris is essential as the crystals are normally seen only when the slit beam is at a certain angle.

Iris crystals are thought to represent unusually large Russell bodies ${ }^{4}$ - spherical, Ig containing structures derived from plasma cells. Russell bodies occur as a result of a block in the normal pathways of Ig secretion within plasma cells. ${ }^{11}$ The sequestration of abnormal Ig within vesicles allows the cell to continue to function. While Russell bodies are a well documented feature of the histopathology of uveitis $^{12}$ they are only rarely seen clinically.

We have documented a series of patients with iris crystals in order to investigate the types of uveitis entity involved, and whether any unifying factors can be identified among them. We have examined the natural history of iris crystals and their response to medical or surgical intervention.

Accepted for publicati

and diagnosis of patients with iris crystals

\begin{tabular}{|c|c|c|c|c|c|c|c|}
\hline $\begin{array}{l}\text { Patient } \\
\text { No }\end{array}$ & $\begin{array}{l}\text { Age crystals } \\
\text { noted (years) }\end{array}$ & $\begin{array}{l}\text { Duration of uveitis before } \\
\text { crystal (months) }\end{array}$ & Sex & Race & Diagnosis & Eye & $\begin{array}{l}\text { Plasma IgG } \\
\text { subclasses }\end{array}$ \\
\hline 1 & 36 & 96 & $M$ & White & Fuchs' heterochromic cyclitis & Right & Normal \\
\hline 2 & 18 & 54 & M & White & Fuchs' heterochromic cyclitis & Left & Normal \\
\hline 3 & 33 & 29 & $\mathrm{~F}$ & White & Fuchs' heterochromic cyclitis & Right & Normal \\
\hline 4 & 38 & 18 & $\mathrm{~F}$ & White & Fuchs' heterochromic cyclitis & Right & Normal \\
\hline 5 & 32 & 216 & $\mathrm{~F}$ & Asian & Fuchs' heterochromic cyclitis & Right & Not done \\
\hline 6 & 77 & 36 & $\mathrm{M}$ & White & Fuchs' heterochromic cyclitis & Right & Not done \\
\hline 7 & 45 & 13 & $\mathrm{~F}$ & White & Fuchs' heterochromic cyclitis & Right & Normal \\
\hline 8 & 30 & 21 & $\mathrm{~F}$ & Asian & Fuchs' heterochromic cyclitis & Right & Not done \\
\hline 9 & 47 & 132 & M & White & Fuchs' heterochromic cyclitis & Left & Normal \\
\hline 10 & 44 & 0 & $\mathrm{~F}$ & White & Fuchs' heterochromic cyclitis & Left & Normal \\
\hline 11 & 27 & 96 & $M$ & White & Fuchs' heterochromic cyclitis & Right & Normal \\
\hline 12 & 21 & 18 & $\mathrm{~F}$ & Afro-Caribbean & Fuchs' heterochromic cyclitis & Left & Raised IgG1 \\
\hline 13 & 37 & 0 & $M$ & White & Fuchs' heterochromic cyclitis & Right & Not done \\
\hline 14 & 81 & 0 & $\mathrm{~F}$ & White & Fuchs' heterochromic cyclitis & Right & Not done \\
\hline 15 & 20 & 0 & $\mathrm{~F}$ & White & Fuchs' heterochromic cyclitis & Left & Normal \\
\hline 16 & 27 & 24 & M & White & Fuchs' heterochromic cyclitis & Left & Not done \\
\hline 17 & 35 & 6 & $M$ & White & Fuchs' heterochromic cyclitis & Right & Not done \\
\hline 18 & 52 & 3 & $\mathrm{~F}$ & White & Chronic idiopathic anterior uveitis & Right & Raised IgG1 \\
\hline 19 & 64 & 60 & M & White & Bilateral idiopathic, chronic anterior uveitis & Right & Not done \\
\hline 20 & 15 & 0.5 & $\mathrm{~F}$ & Asian & Bilateral idiopathic intermediate uveitis & Right & Not done \\
\hline 21 & 28 & 84 & $\mathrm{~F}$ & White & Bilateral idiopathic panuveitis & Right & Normal \\
\hline 22 & 46 & 24 & $\mathrm{~F}$ & White & Bilateral idiopathic panuveitis & Right & Normal \\
\hline 23 & 28 & 3 & $M$ & White & $\begin{array}{l}\text { Bilateral panuveitis and retinal vasculitis. Behçet's } \\
\text { disease }\end{array}$ & Left & Normal \\
\hline 24 & 45 & 180 & $\mathrm{~F}$ & White & Bilateral idiopathic panuveitis and retinal vasculitis & Left & Raised IgG1 \\
\hline
\end{tabular}




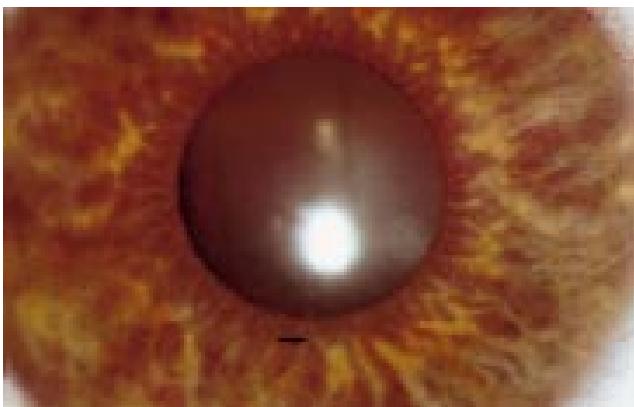

Figure 1

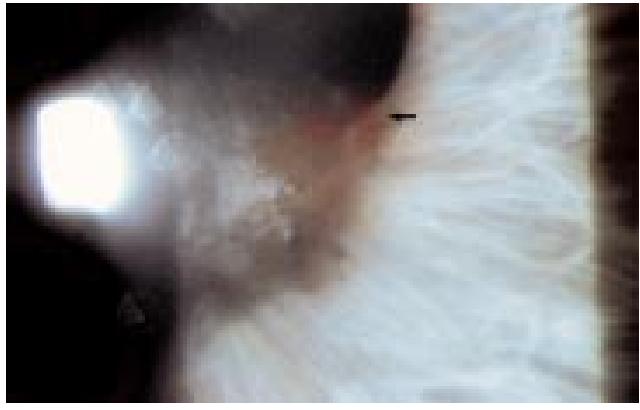

Figure 2

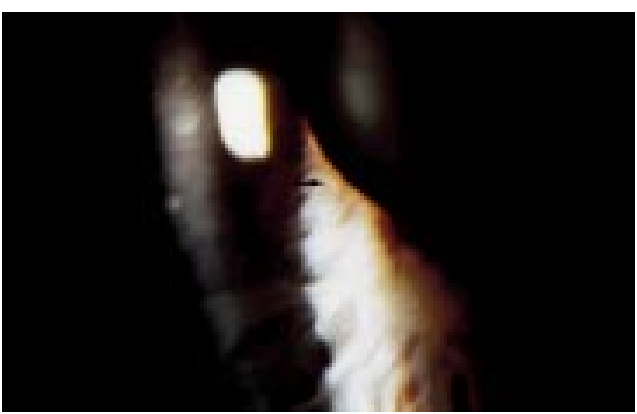

Figure 3

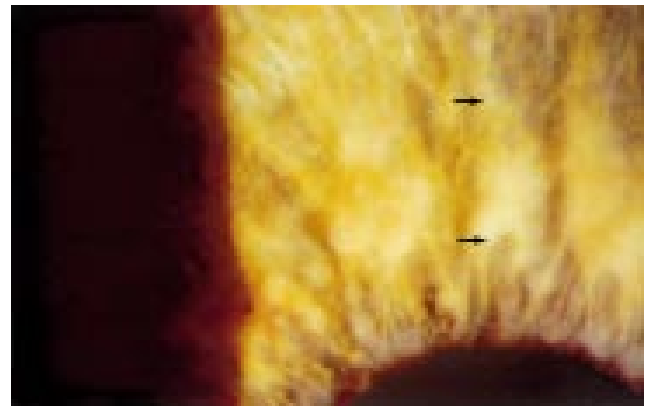

Figure 4

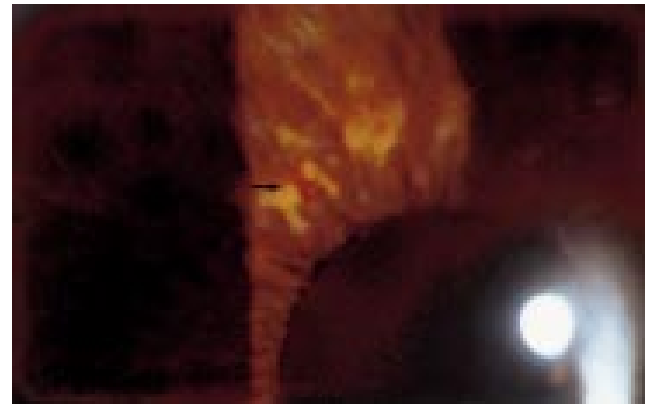

Figure 5

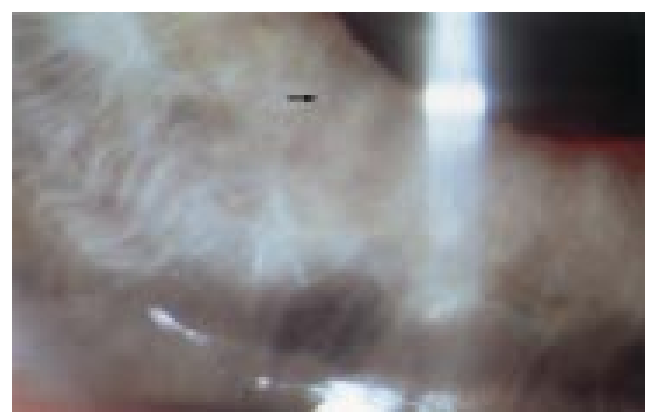

Figure 6

Figures 1-6 Photographs of iris crystals in six patients. Arrows indicate the position of the crystals. The photographs do not do justice to the refractile nature of the crystals.

\section{Materials and methods}

We identified a series of 24 uveitic eyes (24 patients) with iris crystals attending the uveitis clinics of the Birmingham and Midland Eye Centre. Crystals were detected by careful examination under slit lamp biomicroscopy. Photographs of the crystals were taken using a slit lamp anterior segment camera (Kowa SC-6). Demographic details and clinical features of each case were recorded. Retrospective case note review was undertaken to the time when iris crystals were first noted. Patients were then followed prospectively over a 30 month period. The natural history of the iris crystals was documented over a mean follow up period of 15 months.

Following informed consent and approval of the local research ethics committee of the protocol blood samples were taken from 15 patients. Plasma IgG subclasses (IgG1-4) were measured by radial immune diffusion assay.

A histopathological review of all iridectomy specimens obtained in our centre from patients with chronic uveitis over a 3 year period was also undertaken. All 33 patients identified had previously undergone trabeculectomy for un- controlled intraocular pressure. A search was made for evidence of Russell body formation within these specimens.

\section{Results}

There were 14 females and 10 males, with 20 white patients, three Asians, and one AfroCaribbean. The mean age of patients was 38 years (median 36, range 15-81). Seventeen patients had FHC and the other uveitis entities included chronic anterior uveitis, intermediate uveitis, and panuveitis with retinal vasculitis (one patient had Behçet's disease). Demographic data are summarised in Table 1. Six patients had bilateral disease (one chronic anterior uveitis, one intermediate uveitis, and four panuveitis), but there were no cases of bilateral crystals.

Crystals were identified as tiny, glistening, refractile deposits within the iris stroma. They were solitary in nine patients and multiple in 15 patients (range 2-12). Multiple crystals were no more likely in FHC than other diagnoses. Crystals became visible only at certain angles of illumination with the slit lamp 
Table 2 Uveitis entities in 33 iridectomy specimens

\begin{tabular}{lcll}
\hline Uveitis type & $\begin{array}{l}\text { No of } \\
\text { eyes }\end{array}$ & $\begin{array}{l}\text { Number } \\
\text { idiopathic }\end{array}$ & Disease association \\
\hline Chronic anterior uveitis & 7 & 7 & \\
Posner-Schlossmann & 2 & 2 & Rheumatoid arthritis \\
Sclerouveitis & 2 & 0 & \\
Intermediate uveitis & 3 & 3 & Sarcoidosis in 4, V-K-H in 2, Behçet's in 2 \\
FHC & 3 & 3 & 8
\end{tabular}

FHC = Fuchs' heterochromic cyclitis; V-K-H = Vogt-Koyanagi-Harada syndrome.

biomicroscope. Some examples are shown in Figures 1-6.

Crystals were noted on the first visit to a specialist uveitis service in only seven cases. Owing to the minute size of the deposits their presence may have been overlooked. Crystals were first seen approximately 4 years after the onset of symptoms of uveitis (mean 46.4 months, range $0-216$ months, see Table 1 ).

There was no correlation between severity of clinical signs on initial presentation and presence of iris crystals. In some of the patients crystals were first noted when the anterior uveitis was quiescent. In 20 patients, no significant change in the number, size, or position of the iris crystals was seen over the follow up period despite some patients receiving topical and/or systemic therapy. All nine patients requiring treatment (including two with $\mathrm{FHC}$ ) were given topical steroids and cycloplegics. One patient was given a single orbital floor injection of methylprednisolone acetate. Three patients were also given courses of oral prednisolone while under study. One patient was given two courses of antituberculous drugs and another (chronic bilateral panuveitis) took oral cyclosporin.

During the study four patients underwent intraocular surgery and the crystals disappeared immediately following the procedure. They did not reappear over a mean follow up period of 15 months. In three patients (two FHC, one chronic anterior uveitis) cataract surgery was undertaken and in the fourth (with bilateral chronic panuveitis) an iris biopsy was performed to exclude ocular tuberculosis.

Of the 15 patients from whom blood samples were obtained only three had raised levels of plasma IgG1. One of these had FHC, one chronic anterior uveitis, and one chronic panuveitis.

In our review of the histology of iridectomy specimens obtained from patients with chronic uveitis, none showed evidence of Russell body formation. Thirty three specimens were studied with a wide range of uveitis types (Table 2) but only three had FHC.

\section{Discussion}

To our knowledge this is the largest documented series of iris crystals, with 24 eyes of 24 patients. It includes one patient (No 20) previously reported by us. ${ }^{7}$ The majority of patients were white with the most frequent diagnosis being FHC. As 43/428 (10\%) patients attending our uveitis clinics have a diagnosis of FHC, then $17 / 43$ (39.5\%) of FHC patients had evidence of iris crystals.
The first report of iris crystals in a patient with chronic anterior uveitis was published in 1969. ${ }^{4}$ This report included the histopathology of an iridectomy specimen obtained at cataract surgery. A number of large Russell bodies were seen within plasma cells and extracellularly in the superficial iris stroma. These were thought to correspond to the iris crystals seen on clinical examination. Nevertheless, the possibility that these crystals are not Russell bodies, but just free crystals of immunoglobulin cannot be excluded.

Since this report a further 10 cases of iris crystals in patients with chronic uveitis have appeared in the literature. ${ }^{5-10}$ Afro-Caribbean, Asian, and white people were represented. The diagnoses included FHC, sarcoidosis with chronic anterior uveitis, HLA-B27 related chronic anterior uveitis, and panuveitis associated with systemic pseudolymphoma. While uveitis was bilateral in five of these cases crystals were only noted bilaterally in the last. Total plasma immunoglobulin was raised in some, but not all of those tested. Our findings agree with the wide range of diagnoses and racial mix. It is interesting that our series contained a further six cases of bilateral uveitis and none demonstrated bilateral iris crystals. Bilateral crystals appear to be extremely rare.

The natural history of iris crystals suggests that, once formed, they remain clinically apparent and unchanged in position for at least a period of 15 months. Only two of the previously reported cases describe the natural history of iris crystals. In one report, ${ }^{7}$ it is stated that the number of iris crystals in an eye may change from time to time, but no details are given. In another, ${ }^{10}$ the crystals were not reported on two follow up visits but then rediscovered in exactly the same configuration, presumably representing incomplete record taking.

In our series therapeutic intervention with topical and systemic drugs had no effect on their appearance. Surgical intervention may lead to disappearance of the crystals, perhaps through mechanical disruption from the force of intraocular irrigation or perhaps they were dissolved in the intraocular fluids used in surgery. Nevertheless, attempts to dislodge crystals for laboratory analysis were fruitless as they appeared firmly adherent to the iris. Iris biopsy by iridectomy during cataract surgery in the three patients in this series was considered unethical.

Russell bodies are known to occur in many types of chronic inflammation including chronic uveitis, and in various B lymphocyte tumour lines. ${ }^{11}$ They are thought to represent the sequestration of an abnormal Ig which can neither exit from, nor be degraded in, the endoplasmic reticulum. ${ }^{12}$ The accumulation of these abnormal molecules within intracellular vesicles allows the cell to survive. Thus the presence of Russell bodies within the eye is evidence of active immunoglobulin secretion. Raised aqueous Ig levels have been reported in uveitis $^{2}$ and oligoclonal Ig (mainly IgG1) has been found in FHC. ${ }^{23}$ If Ig production is a frequent feature of FHC it may explain why this 
condition is so common in our series in spite of FHC accounting for approximately $5 \%$ of all uveitis entities.

Patient number 21 was interesting. This was a woman with a recurrent right iris mass which dramatically responded to two courses of antituberculous therapy. When the mass reappeared for the third time an iris biopsy was performed but the histology failed to reveal a definitive diagnosis. Mycobacterial DNA was also not detected in the aqueous using the polymerase chain reaction. The mass subsequently responded to a course of systemic steroid.

Studies of plasma Ig levels in anterior uveitis, and specifically in FHC give uncertain results $^{13-15}$ perhaps because ocular inflammation is too localised to cause changes in humoral antibody concentration. Our analysis of plasma IgG subclasses in patients with crystals was also unhelpful as plasma IgG1 was raised in the minority of cases with no predilection for patients with FHC.

Iris crystals are a fascinating clinical sign seen in chronic intraocular inflammation. They are found more often in patients with FHC than in other forms of chronic uveitis. Once formed, the crystals rarely disappear and medical treatment has little impact on their presence, nor does the degree of disease activity. Surgical intervention may result in the disappearance of crystals as early as the first postoperative day, possibly as a result of mechanical disruption.

Although they appear to be rare, the minute size of the lesions may result in underreporting. They may well be missed if insufficient time is given to examination of the iris. We gained the impression that our rate of discovery of new patients increased with our awareness of this sign.

Russell bodies are recognised as established features of the histopathology of chronic uveitis, ${ }^{16}{ }^{17}$ but our observations suggest that they are unusual, as a review of the histopathology of 33 iridectomy specimens failed to demonstrate a single Russell body.
Uveitis is perhaps the most puzzling of all ocular diseases. The underlying pathology remains ill understood and Russell body formation is merely a reflection of plasma cell activity within the iris stroma. Thus, their presence does not appear to shed further light on the mechanisms underlying the pathogenesis of specific uveitis entities. Nevertheless, we recommend that a careful search for iris crystals is undertaken in every case of uveitis to document this remarkable clinical sign.

1 Dick AD. Experimental approaches to specific immunotherapies in autoimmune disease: future treatment of endogenous posterior uveitis. $\mathrm{Br} \mathcal{F}$ Ophthalmol 1996; 103:96-104

2 Murray PI, Hoekzema R, Luyendijk L, et al. Analysis of aqueous humor immunoglobulin $\mathrm{G}$ in uveitis by enzymelinked immunosorbent assay, isoelectric focusing, and immunoblotting. Invest Ophthalmol Vis Sci 1990;31:212935.

3 Murray PI, Hoekzema R, Van Haren MAC, et al. Aqueous humour analysis in Fuchs' heterochromic cyclitis. Curr Eye Res 1990;9(suppl):53-7.

4 Iwamoto T, Witmer R. Light and electron microscopy on plasma cells and Russell bodies in the iris of a chronic uveitis patient. Invest Ophthalmol Vis Sci 1969;8:563-82.

5 Lam S, Tessler HH. Iris crystals and hypergammaglobulinaemia. Am f Ophthalmol 1990;110:440-1.

6 Lam S, Tessler HH, Winchester K, et al. Iris crystals in chronic iridocyclitis. Br f Ophthalmol 1993;77:181-2.

7 Murray PI. Iris crystals and uveitis. $\mathrm{Br} f$ Ophthalmol 1993;77:466.

8 Meyer E, Lichtig C, Golan DT, et al. Shiny spots in the iris in chronic uveitis. F Pediatr Ophthalmol Strabismus 1980;17: 305-6.

9 Zamir E, Margalit E, Chowers I. Iris crystals in Fuchs' heterochromic iridocyclitis. Arch Ophthalmol 1998;116:1394.

10 Bialasiewicz A, Gierth K, Nauman GOH. Heterochromiacomplicata Fuchs, crystalline iridopathy and elevation of IgG concentration in aqueous humour. Klin Monatsbl Augenheilkd 1991;198:205-6.

11 Hsu SM, Hsu PL, McMillan PN, et al. Russell bodies, a light and electron microscopic immunoperoxidase study. Am 7 Clin Path 1982;77:26-31.

12 Valetti C, Grossi CE, Milstein C, et al. Russell bodies: a general response of secretory cells to synthesis of a mutant immunoglobulin which can neither exit from, nor be degraded in the endoplasmic reticulum. $f$ Cell Biol 1994;115:983-4.

13 Norn MS. Immunoglobulins in endogenous uveitis. $\mathrm{Br} f$ Ophthalmol 1976;60:299-301

14 Kahn LS, Schlaegel TF, Weber JC, et al. Serum immunoglobulins and uveitis. Arch Ophthalmol 1983;101:458-9. 5 McCoy R, White L, Tait B, et al. Serum immunoglobulins in acute anterior uveitis. Brf Ophthalmol 1984;68:807-10.

16 Howes EL. Basic mechanisms in pathology. In: Spencer WH, ed. Ophthalmic pathology: an

17 Yanoff M, Fine BS. Nongranulomatous inflammation: uveitis, endophthalmitis, panophthalmitis, and sequelae. Ocular pathology. 4th ed. New York: Mosby-Wolfe, 1996:59. 